\title{
Stage II Uterine Corpus Leiomyosarcoma AJCC v8
}

National Cancer Institute

\section{Source}

National Cancer Institute. Stage II Uterine Corpus Leiomyosarcoma A/CC v8. NCI

Thesaurus. Code C139874.

Stage II includes: T2, N0, M0. T2: Uterine corpus leiomyosarcoma with tumor extending beyond the uterus, within the pelvis. NO: No regional lymph node metastasis. MO: No distant metastasis. (AJCC 8th Ed.) 